\title{
Application of uncertainty and sensitivity analysis to the air quality SHERPA modelling tool
}

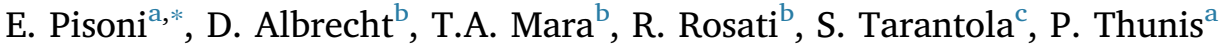 \\ a European Commission, Joint Research Centre (JRC), Directorate for Energy Transport and Climate, Air and Climate Unit, Via E. Fermi 2749, I-21027, Ispra, VA, Italy \\ ${ }^{\mathrm{b}}$ European Commission, Joint Research Centre (JRC), Directorate I - Competences - Modelling, Indicators \& Impact Evaluation Unit, Via E. Fermi 2749, I-21027, Ispra, \\ VA, Italy \\ ${ }^{\mathrm{c}}$ European Commission, Joint Research Centre (JRC), Directorate for Energy Transport and Climate, Energy Security Distribution and Markets Unit, Via E. Fermi 2749, I- \\ 21027, Ispra, VA, Italy
}

\section{A R T I C L E I N F O}

\section{Keywords:}

Uncertainty analysis

Sensitivity analysis

Air quality modelling

Surrogate models

Model quality assurance

\begin{abstract}
A B S T R A C T
Air quality has significantly improved in Europe over the past few decades. Nonetheless we still find high concentrations in measurements mainly in specific regions or cities. This dimensional shift, from EU-wide to hotspot exceedances, calls for a novel approach to regional air quality management (to complement EU-wide existing policies). The SHERPA (Screening for High Emission Reduction Potentials on Air quality) modelling tool was developed in this context. It provides an additional tool to be used in support to regional/local decision makers responsible for the design of air quality plans. It is therefore important to evaluate the quality of the SHERPA model, and its behavior in the face of various kinds of uncertainty. Uncertainty and sensitivity analysis techniques can be used for this purpose. They both reveal the links between assumptions and forecasts, help inmodel simplification and may highlight unexpected relationships between inputs and outputs.

Thus, a policy steered SHERPA module - predicting air quality improvement linked to emission reduction scenarios - was evaluated by means of (1) uncertainty analysis (UA) to quantify uncertainty in the model output, and (2) by sensitivity analysis (SA) to identify the most influential input sources of this uncertainty. The results of this study provide relevant information about the key variables driving the SHERPA output uncertainty, and advise policy-makers and modellers where to place their efforts for an improved decision-making process.
\end{abstract}

\section{Introduction}

Air quality has significantly improved in Europe over the past few decades (EEA, 2017), but exceedances of the legislative limit values still persist, mainly for pollutants such as ozone $\left(\mathrm{O}_{3}\right)$, nitrogen dioxide $\left(\mathrm{NO}_{2}\right)$ and particulate matter (PM10 and PM2.5) ${ }^{1}$. While, in the past years, these exceedances were wide-spread across Europe, they now tend to concentrate in specific regions or cities (Kiesewetter et al., 2015). This new and changed situation calls for a novel approach tailored to local air quality management (to complement EU-wide existing policies).

There is a long standing tradition of using modelling techniques in supporting the design of air quality policies. A first set of techniques consists of three dimensional numerical models that simulate transport, chemistry, emissions, and deposition in the atmosphere (Mailler et al., 2016; Pernigotti et al., 2013). Given their complexity and demanding/ onerous requirements (in terms of data preparation, scientific/technical knowledge and computing time), these models are mainly used for scientific research. For such models, state-of-the art approaches are available to compute sensitivity coefficients measuring how the concentrations predicted by the model depend on input data and model parameters. These approaches vary from conceptually simple ones, as the brute-force (varying the input parameters one by one in separate model simulations and evaluating the change in predicted concentrations) to more complex, as decoupled direct method and the adjoint method (Dunker et al., 2002; Sandu et al., 2003; Kelly et al., 2015). All these methods are usually applied to a fully-fledged air quality model, to perform its local sensitivity analysis.

In addition to three dimensional numerical models, another set of approaches has been developed, mainly to deal with the 'science-topolicy' interface. These approaches are referred to as "Integrated Assessment Models", as they integrate various dimensions: policy costs, benefits, etc ... in one single approach. In such type of approaches, the air quality component is not based on the full air quality model

\footnotetext{
* Corresponding author.

E-mail address: enrico.pisoni@ec.europa.eu (E. Pisoni)

${ }^{1}$ PM: inhalable particles, with diameters that are 10 or 2.5 micrometers.
} 
previously mentioned (that would be too time consuming to be simulated) but usually it is implemented as a "surrogate" of the full air quality model. A valuable example of "Integrated Assessment Model" is the GAINS-EU (Greenhouse Gas - Air Pollution Interactions and Synergies) integrated assessment model (Amann et al., 2011), which is based on linear source-receptor relationships to link emissions to concentrations, and has frequently been used to choose optimal emission reductions per country, in order to achieve environmental improvements at a minimum cost. In the last years, given the current situation marked by regional and/or local (city) hot spots, the EU integrated assessment modelling tools have also been complemented/aided by both regional and local approaches. This has been done in recent years using national versions of GAINS based on finer scale modelling (as in GAINS-Italy, D'Elia et al., 2009), or with regional tools (as RIAT, the Regional Integrated Assessment Tool, Carnevale et al., 2012, Carnevale et al., 2014, Pisoni et al., 2010). These efforts have already supported the implementation of regional/local plans, but it is important to bear in mind that their application heavily relies on the availability of detailed local data and of complex scientific/technical know-how, not always readily available on a local scale.

Recently, the SHERPA (Screening for High Emission Reduction Potentials on Air quality) modelling tool was developed (Clappier et al., 2015; Thunis et al., 2016; Pisoni et al., 2017) to provide an alternative approach. SHERPA, which is based on a "surrogate model" replicating the behavior of a fully-fledged air quality model, serves as a tool to support regional/local decision makers responsible for the design of air quality plans. It is distributed with default data that covers the whole Europe and enables decision-makers to work on their own regional domain. It can be used without the need to perform prior complex scientific/technical tasks. SHERPA supports decision-makers who need to plan air quality policies by implementing modules such as "source allocation" (to apportion air pollution in terms of sectors and precursors of origin), "governance" (to identify the key geographical entities contributing to the pollution in one specific area), "scenario" (to test the effect on air quality of a given sector-specific emission abatement scenario). As the tool will be used in the policy arena, it is of utmost importance to evaluate the robustness of the model predictions with regards to various sources of uncertainty.

Uncertainties can be particularly influential in policy context. It is widely known that model and data are uncertain and that uncertainties may be very significant. It is therefore important to know how model outputs, namely potential policy impacts are affected by these uncertainties. The uncertainty quantification process helps to understand whether models are "fit for the purpose" and/or apt to be used in the field of policy making. Complementary to this, sensitivity analysis (SA) should also be applied. While the uncertainty analysis (UA) aims at quantifying uncertainty in the model output, sensitivity analysis investigates the dependency of the model output from various sources of uncertainty in the model inputs (Saltelli et al., 2008). Sensitivity analysis is an important ingredient in the quality assurance of models used for evidence-based policy and, because it reveals the links between assumptions and predictions, it helps in model simplification (i.e. not relevant input can be identified) and model calibration (i.e. optimal parameters setting). It can highlight unexpected relationships between inputs and outputs, helping to identify regions of the input space which are responsible for critical values of the output.

In this paper, we perform the uncertainty and sensitivity analysis of the SHERPA "scenario" module (Thunis et al., 2016). This module allows for the estimation of how concentrations change due to various given emission-reduction scenarios. It is used as a basis for all SHERPA modules and is therefore the key element to be tested. As SHERPA is a model characterized by spatially-varying coefficients and inputs, the Uncertainty and Sensitivity Analysis (UA-SA) have been performed on a few selected cities (Helsinki, Constanța, London, and Milan, see Fig. 1), representative of different meteorological and of varying emission inventory conditions (the same analysis is presented in Albrecht et al.,
2018 on an extended set of cities, showing similar conclusions; so here, for lack of space, we focus on a smaller number of cities). This analysis focuses on two main issues: (1) what is the robustness level of the SHERPA results in terms of uncertainty of the model response (uncertainty analysis) and (2) how the model output is influenced by each model input - parameters, precursors, and policy choices (sensitivity analysis).

In this study we answer these research questions within a Global Sensitivity Analysis (GSA) variance-based framework. As opposed to local sensitivity analysis (i.e. with brute-force, decoupled direct and adjoint methods, previously discussed), GSA measures the relative importance of the model inputs by exploring the entire input space. In particular, GSA has been carried out using the popular methods described in Saltelli et al. (2010). The results provide information about the key variables driving the SHERPA output uncertainty. This paper is organized as follows: In Section 2, we briefly introduce the SHERPA model and the sensitivity analysis method employed to analyze it. In Section 3, we define the model input uncertainties. Furthermore, we discuss the results in Section 4 before reaching our conclusion in Section 5 .

\section{Materials and methods}

In this chapter the SHERPA model (both formalization and its assumptions/caveats) and the technique used to evaluate uncertainty and sensitivity analysis are presented.

\subsection{The Sherpa model}

SHERPA has been developed to provide a speedy modelling approach to calculate concentration fields resulting from emission reduction scenarios, mimicking the behavior of a full Chemical Transport Model (CTM). CTMs provide pollutant concentration fields that account for the complex transport, diffusion and chemical processes occurring in the atmosphere. The aim of SHERPA is to mimic CTMs' behavior with a simpler relationship/equation derived from a set of full CTM simulations built with various emission reduction scenarios. This set of scenarios should be sufficiently varied (in terms of concentration changes, responses to emission changes) to provide the SHERPA training phase with sufficient data variability.

In SHERPA, concentration changes due to an emission reduction scenario are computed on a cell by cell basis according to the following equation:

$\Delta \mathrm{C}_{\mathrm{n}}=\sum_{\mathrm{p}}^{\mathrm{N}_{\mathrm{prec}}} \sum_{\mathrm{m}}^{\mathrm{N}_{\text {cell }}} \mathrm{a}_{\mathrm{n}, \mathrm{p}, \mathrm{m}} \Delta \mathrm{E}_{\mathrm{p}, \mathrm{m}}, \forall n \in\left[1, N_{\text {cell }}\right]$

where the delta concentration $\Delta \mathrm{C}_{\mathrm{n}}$ (change of concentration in comparison to the base case) in a receptor grid cell " $n$ " is expressed as a linear combination of the emissions delta $\Delta \mathrm{E}_{\mathrm{p}, \mathrm{m}}$ (variation in emission when compared to the base case), for each source cell " $\mathrm{m}$ " and pollutant (i.e. precursor) "p". The $a_{n, p, m}$ coefficients act as weighting factors which apportion the amount of emission variation $\Delta \mathrm{E}_{\mathrm{p}, \mathrm{m}}$ of precursor $\mathrm{p}$ stemming from cell $\mathrm{m}$ and reaching cell $\mathrm{n}$. As the correlation between $\Delta \mathrm{C}_{\mathrm{n}}$ (at receptor cell $\mathrm{n}$ ) and $\Delta \mathrm{E}_{\mathrm{p}, \mathrm{m}}$ (at all sources cell $\mathrm{m}$ ) decreases with the distance between the cells, it has been assumed that the coefficients $\mathrm{a}_{\mathrm{n}, \mathrm{p}, \mathrm{m}}$ in the previous equation can be approximated by the following distance-function:

$\mathrm{a}_{\mathrm{n}, \mathrm{p}, \mathrm{m}}=\alpha_{\mathrm{n}, \mathrm{p}}\left(1+\mathrm{d}_{\mathrm{n}, \mathrm{m}}\right)^{-\omega^{\mathrm{n}, \mathrm{p}}}$

where $d_{n, m}$ is the distance between cells $n$ and $m$ and the two unknowns $\alpha$ and $\omega$ for each precursor $p$ and each grid cell $n$ were estimated from CTM simulation results (see Pisoni et al., 2017 for more details).

Even though the previous equations remain the same/unvaried everywhere in the whole calculation domain, the values of $\alpha$ and $\omega$ are grid-cell specific. The parameter $\alpha$ is related to the amplitude of the 


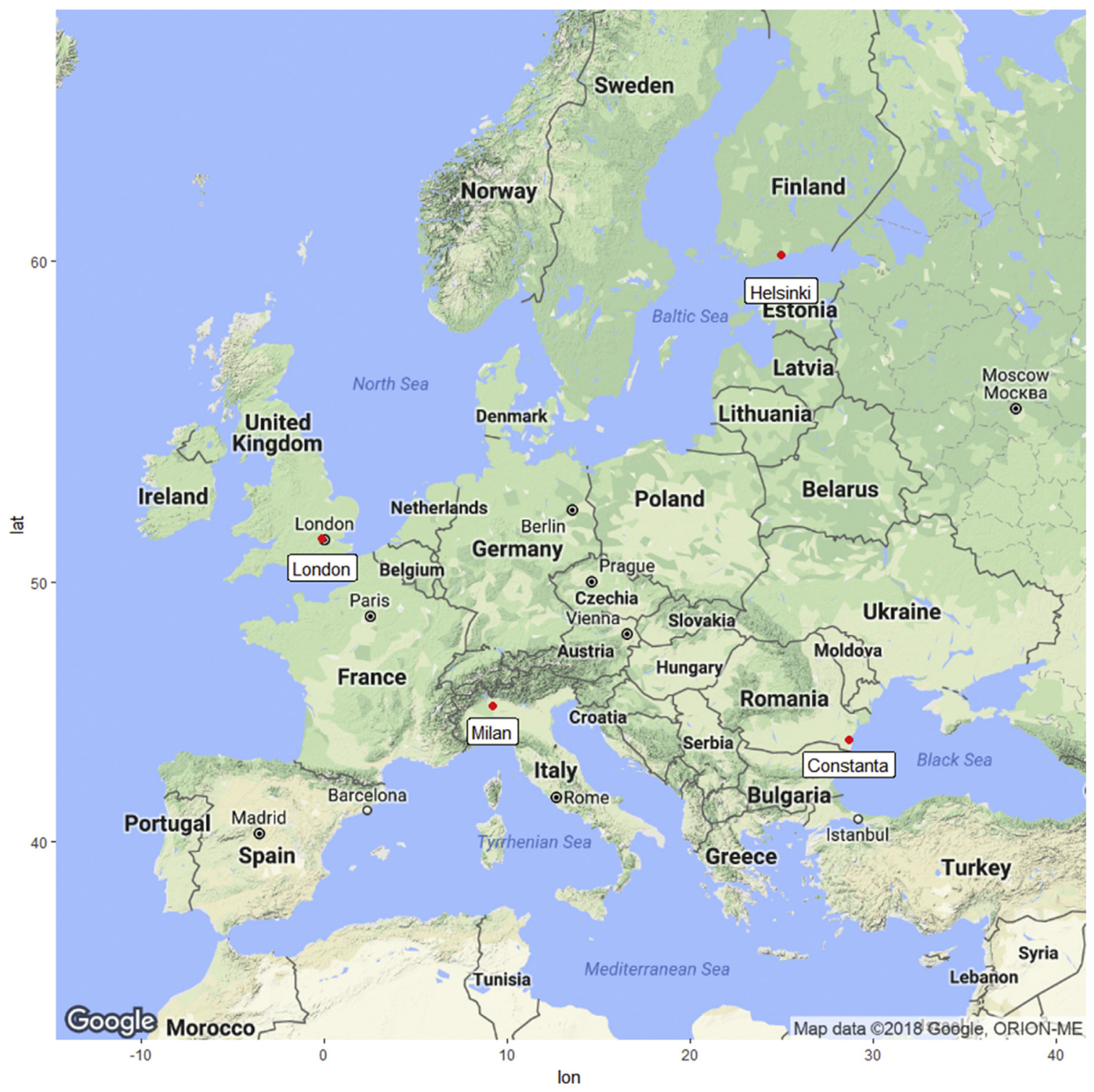

Fig. 1. Map of Europe, showing the location of the four selected cities.

function and provides information about the relative importance of an emission precursor with respect to another, whereas $\omega$ provides information on the speed of decrease of the emissions impact with distance. The $\omega$ parameter depends on meteorological conditions, especially wind speed, and is also precursor specific (some emission precursors have longer lifetime spans in the atmosphere). Both $\alpha$ and $\omega$ were identified through a least-square estimation, using data provided by CTM simulations. This identification process also provided confidence intervals for these coefficients. More details on the procedure can be found in Clappier et al. (2015), Thunis et al. (2016), and Pisoni et al. (2017).

After $\alpha$ and $\omega$ have been computed, SHERPA can be used to evaluate concentration changes resulting from any emission reduction scenario. But because these coefficients were obtained with confidence intervals, it is important to account for the impact of these uncertainties on SHERPA's outputs. The current SHERPA implementation (Pisoni et al., 2017), which is able to simulate PM10, PM25 and $\mathrm{NO}_{2}$ yearly averages, will be used to perform the uncertainty and sensitivity analysis presented in this paper, in particular, focusing on PM2.5.

\subsection{Caveats about the SHERPA model}

On top of the uncertainty and sensitivity analysis performed in the next sections, there are a number of assumptions/caveats in the SHERPA model. These should be taken into account when evaluating the model results, as they can affect the quality of the results.

\subsubsection{Link with the full air quality model}

The SHERPA results strongly depend on the air quality model used to define its coefficients, in the training and validation phases. In the configuration presented in this paper, SHERPA is based on the CHIMERE model results. Although the CHIMERE CTM base case scenario, in a similar configuration, has been extensively validated against observations (Bessagnet et al., 2016), it is not possible to validate CTMs for model-responses to emission changes. This is the reason why CTMs are regularly tested in the frame of inter-comparison exercises (Cuvelier et al., 2007). This process of inter-comparison is on-going, to increase the reliability and robustness of the whole approach.

\subsubsection{Spatial resolution}

The CHIMERE simulations within SHERPA are run with a $7 \mathrm{~km}$ spatial resolution. Schaap et al. (2015) showed that this resolution was 
accurate enough to capture urban background concentrations, the focus of this work. This spatial resolution however limits the analysis to urban background pollution, and so prevent the model to be used e.g. for reproducing traffic station behavior.

\subsubsection{Meteorological variability}

The results presented in this article are based on a single meteorological year (2009). Although this year is thought to be representative of average meteorological conditions, the current set-up does not account for inter-annual variability.

\subsubsection{Linearity assumption}

The relationship between emission and concentration used in SHERPA is assumed as linear. Given the non-linear chemical processes leading to the formation of secondary PM, we expect non-linear relationships between PM concentrations and their precursor emissions. In their work, Thunis et al. (2015) have shown that these relationships can be assumed linear when concentrations are averaged over long periods (seasonal or annual averages). It is important to note, however, that these tests were performed for emission reductions not exceeding $50 \%$ reduction. Beyond these levels, non-linearity might become more important, limiting the range of application of the approach.

\subsubsection{The "bell-shape" assumption}

A simple "bell shape" function spatially linking emission and concentration changes is used in SHERPA. Statistical analysis shows that the correlations between PM concentrations and their precursor emissions decrease with distance following a "bell shape" function (Pisoni et al., 2017). So, this "bell shape" function is used to express the coefficients of the SRR as a function of the distance between sources and receptors. This simple "bell shape" function is precursor and grid cell specific. These assumptions (with the linearity one previously presented) have been assessed through a validation process, which covered test cases in many EU cities, regions and countries. This validation highlighted the good agreement between the SHERPA SRR and the full CHIMERE model (Pisoni et al., 2017).

\subsubsection{Point sources and area sources treatment}

SHERPA distinguishes sectoral impacts, but point sources (for which the release height becomes an important element) and surface sources are treated similarly. This might introduce some uncertainty in the model behavior (i.e. model inadequacy) when both low- and high-level emission sources are present in the same sector.

\subsection{Variance -based sensitivity indices}

In this section we briefly explained the indicators for sensitivity analysis used in this paper.

Historically, UA-SA studies were firstly conducted by using local approaches. In the local analysis, inputs (or model parameters) are varied 'One at A Time' (OAT) while the others are maintained fixed. This kind of approach implies calculation or estimation of partial (often normalized) derivatives of the model output at a given point in the input space. The local techniques are computationally cheap, however, (i) they are not fit for non-linear models, and (ii) the possible effects deriving from the interactions between different model inputs are ignored. Therefore, local SA is definitely perfunctory (Saltelli and Annoni, 2010) and not recommended when models are not linear and/or not merely additive (Campolongo and Saltelli, 1997).

Global sensitivity analysis overcomes the drawback of the OAT approach making use of methods based on the simultaneous exploration of all uncertain inputs and thus being able to capture nonlinearities and interactions among model inputs. GSA allows for full exploration of the input space, in order to exhaustively assess any output uncertainty. Furthermore, compared with derivative-based SA, GSA is more effective at protecting against type II errors ${ }^{2}$ and provides more insight into the model input-output relationship. However, often GSA requires many model runs.

Since the early 90s, GSA has been developing in, and applying to a wide range of domains (i.e. Environment, Engineering, Medicine, Chemistry, ...), showing itself to be central in contributing to modelling (Tarantola et al., 2002). Different methods are available to conduct sensitivity analysis nowadays (Sobol, 2001; Borgonovo, 2007; Liu and Homma, 2009; Saltelli et al., 2010; Mara and Tarantola, 2012; Kucherenko et al., 2012; Plischke et al., 2013). Among them, variance-based methods are very popular and often considered the most powerful.

Variance-based techniques (used in this paper) rely on the decomposition of the total variance of the model output $Y=f(X)$ into the sum of partial variances due to/resulting from the uncertain model inputs $\mathbf{X}=\left(\mathrm{X}_{1}, \ldots, \mathrm{X}_{\mathrm{d}}\right)$ (Sobol, 1993). The model inputs are treated as independent random variables, each of them defined by their marginal probability distribution. The method of Sobol (1993) enables the computing of the terms in the variance decomposition in a quite intuitive way by estimation of a multidimensional integral via Monte Carlo techniques:

$V(Y)=\sum_{\mathrm{i}=1}^{\mathrm{d}} \mathrm{V}_{\mathrm{i}}+\sum_{\mathrm{j}>\mathrm{i}}^{\mathrm{d}} \mathrm{V}_{\mathrm{i}, \mathrm{j}}+\cdots+\mathrm{V}_{1, \ldots, \mathrm{d}}$

where $\mathrm{V}(\mathrm{Y})$ is the total variance of the model output $\mathrm{Y}$. This equation is the so-called ANOVA decomposition that casts the total variance of the model response onto summands of all the possible effects, which are the first-order effects $V_{i}$, the second-order effects $V_{i j}$ that measure the joint effect of the pair $\left(\mathrm{X}_{\mathrm{i}}, \mathrm{X}_{\mathrm{j}}\right)$ on $\mathrm{Y}$, and the higher order interactions $\mathrm{V}_{1,2, \ldots, \mathrm{d} \cdot}$.

It can be shown that the variance of the conditional expectation $\mathrm{V}\left(\mathrm{E}\left(\mathrm{Y} \mid \mathrm{X}_{\mathrm{i}}\right)\right)$ is equal to the partial variance $\mathrm{V}_{\mathrm{i}}$. This statistic is known as the first-order, or main effect of model input $\mathrm{X}_{\mathrm{i}}$ on the model output $\mathrm{Y}$. It corresponds to the contribution of $\mathrm{X}_{\mathrm{i}}$ by itself to the total variance of Y. This partial variance, divided by the total variance of the model output, produces a normalized value, which defines the sensitivity index of $X_{i}$, called first-order index of the input $X_{i}$ :

$\mathrm{S}_{\mathrm{i}}=\frac{\mathrm{V}\left(\mathrm{E}\left(\mathrm{Y} \mid \mathrm{X}_{\mathrm{i}}\right)\right)}{\mathrm{V}(\mathrm{Y})}$

where $\mathrm{E}(.$. . ) stands for the conditional expectation operator.

$S_{i}$ is, by definition, a number between 0 and 1 . A high value of $S_{i}$ denotes an influential input, in the sense that the uncertainty of the input $X_{i}$ has a considerable effect on the uncertainty of the model output $\mathrm{Y}$.

By noting that an input can contribute solely or by interactions with other inputs, Homma and Saltelli (1996) introduce the total sensitivity index of a model input as the sum of all the terms of any order involving that input. Being the sum of all possible partial variances equal to $\mathrm{V}(\mathrm{Y})$ (see Eq. (3)), the difference between $\mathrm{V}(\mathrm{Y})$ and $\mathrm{V}\left(\mathrm{E}\left(\mathrm{Y} \mid \mathbf{X}_{\sim \mathrm{i}}\right)\right.$ ) - which expresses all terms of any order that do not include input $X_{i}(\sim i$ indicates all terms but $\mathrm{i}$ ) - represents the total effect of input $\mathrm{X}_{\mathrm{i}}$. Therefore, the total sensitivity index is defined as follows:

$\mathrm{T}_{\mathrm{i}}=1-\frac{\mathrm{V}\left(\mathrm{E}\left(\mathrm{Y} \mid \mathbf{X}_{\sim \mathrm{i}}\right)\right)}{\mathrm{V}(\mathrm{Y})}$

Further, given the law of total variance:

$\mathrm{V}(\mathrm{Y})=\mathrm{E}\left(\mathrm{V}\left(\mathrm{Y} \mid \mathrm{X}_{\mathrm{i}}\right)\right)+\mathrm{V}\left(\mathrm{E}\left(\mathrm{Y} \mid \mathrm{X}_{\mathrm{i}}\right)\right)$

the total sensitivity index of $\mathrm{X}_{\mathrm{i}}$ can also be defined as:

$\mathrm{T}_{\mathrm{i}}=\frac{\mathrm{E}\left(\mathrm{V}\left(\mathrm{Y} \mid \mathbf{X}_{\sim \mathrm{i}}\right)\right)}{\mathrm{V}(\mathrm{Y})}$

${ }^{2}$ The risk of declaring non important an input which is actually important 
Table 1

Mean and standard deviations (Std) of the normal distributions associated with the coefficients $\alpha$ and $\omega$ for different cities.

\begin{tabular}{|c|c|c|c|c|c|c|c|c|}
\hline \multirow[t]{2}{*}{ Coefficient } & \multicolumn{2}{|l|}{ Helsinki } & \multicolumn{2}{|c|}{ Constanța } & \multicolumn{2}{|l|}{ London } & \multicolumn{2}{|l|}{ Milan } \\
\hline & Mean & Std & Mean & Std & Mean & Std & Mean & Std \\
\hline$\omega_{-} \mathrm{NOx}$ & 1.8681 & 0.0104 & 1.9728 & 0.0221 & 1.8681 & 0.0104 & 1.9728 & 0.0221 \\
\hline$\omega_{-} \mathrm{NH}_{3}$ & 1.6201 & 0.0094 & 1.6005 & 0.0202 & 1.6201 & 0.0094 & 1.6005 & 0.0202 \\
\hline$\omega_{-} \mathrm{ppm}$ & 2.5056 & 0.0066 & 2.3294 & 0.0173 & 2.5056 & 0.0066 & 2.3294 & 0.0173 \\
\hline$\omega_{-} \mathrm{SO}_{2}$ & 1.3761 & 0.0105 & 1.3397 & 0.0091 & 1.3761 & 0.0105 & 1.3397 & 0.0091 \\
\hline$\alpha \_\mathrm{NOx}$ & 0.0032 & 0.0004 & 0.0494 & 0.0041 & 0.0109 & 0.0006 & 0.0497 & 0.0050 \\
\hline$\alpha \_\mathrm{NH}_{3}$ & 0.1365 & 0.0054 & 0.0680 & 0.0039 & 0.0704 & 0.0031 & 0.0679 & 0.0133 \\
\hline a_ppm & 0.4994 & 0.0087 & 0.5332 & 0.0133 & 0.2804 & 0.0338 & 1.9693 & 0.0422 \\
\hline$\alpha \_S_{2}$ & 0.0026 & 0.0004 & 0.0065 & 0.0004 & 0.0023 & 0.0005 & 0.0123 & 0.0048 \\
\hline
\end{tabular}

There are several numerical methods proposed in the literature to estimate the first-order and total sensitivity indices (to name a few, Saltelli et al., 1999; Sobol, 2001; Saltelli, 2002; Buzzard and Xiu, 2011; Shao et al., 2017, Blatman and Sudret, 2010). In the present study, we use the sampling-based strategy proposed in Saltelli (2002). This method requires generating two independent samples $\mathbf{A}$ and $\mathbf{B}$ of $\mathbf{X}$. These samples represent two matrices of size $\mathrm{N}$ by $\mathrm{d}$ that contain in their ith column random draws of $\mathrm{X}_{\mathrm{i}}$ sampled from its marginal distribution. Thus, each row of $\mathbf{A}$ and $\mathbf{B}$ is employed to carry out Monte Carlo runs of the model. This provides two vectors of model responses $f\left(\mathbf{A}_{\mathbf{j}}\right)$ and $f\left(\mathbf{B}_{\mathrm{j}}\right), \mathbf{j}=1, \ldots, \mathrm{N}$ from which the total variance can be estimated (denoted $\hat{V}_{Y}$ ). Furthermore, d additional Monte Carlo samples $\mathbf{A}^{\mathbf{B}, \mathbf{i}}$ with $\mathbf{i}=1, \ldots, d$, are also required. The sample $\mathbf{A}^{\mathbf{B}, \mathbf{i}}$ is equal to $\mathbf{A}$ except the ith column replaced by the ith column of $\mathbf{B}$. These samples $d$ are also propagated throughout the model to obtain $f\left(\mathbf{A}_{\mathrm{j}}^{\mathbf{B}, \mathrm{i}}\right)$. Finally, the variance-based sensitivity indices are estimated as follows:

$\hat{\mathrm{S}}_{\mathrm{i}}=\frac{\frac{1}{\mathrm{~N}} \sum_{\mathrm{j}=1}^{\mathrm{N}} f\left(\mathbf{B}_{\mathrm{j}}\right)\left[f\left(\mathbf{A}_{\mathrm{j}}^{\mathbf{B}, \mathrm{i}}\right)-f\left(\mathbf{A}_{\mathrm{j}}\right)\right]}{\hat{\mathrm{V}}_{\mathrm{Y}}}$

$\hat{\mathrm{T}}_{\mathrm{i}}=\frac{\frac{1}{2 \mathrm{~N}} \sum_{\mathrm{j}=1}^{\mathrm{N}}\left[f\left(\mathbf{A}_{\mathrm{j}}^{\mathbf{B}, \mathrm{i}}\right)-f\left(\mathbf{A}_{\mathbf{j}}\right)\right]^{2}}{\hat{\mathrm{V}}_{\mathrm{Y}}}$

These definitions and procedures related to sensitivity indices were applied to our study of the SHERPA model.

\section{Characterization of sources of uncertainty}

\subsection{The general setting}

To study the uncertainty affecting the model, we take all quantifiable uncertainties into account, and as derived from each input of the model. Generally speaking, these uncertainties might be estimated starting from the probability distributions of the variables involved.

Given our particular case, it is necessary to identify all the terms affecting the air quality modelled at a city level (as aforementioned earlier, PM2.5 yearly averages), that are:

- The model coefficients: four values for $\alpha$ and four values for $\omega$, defining the link between precursor emissions $\left(\mathrm{NO}_{\mathrm{X}}\right.$ nitrogen oxides, $\mathrm{NH}_{3}$ ammonia, PPM primary particulate matter, $\mathrm{SO}_{2}$ sulphur dioxide) and concentrations;

- The precursor emission inputs (again, related to $\mathrm{NO}_{\mathrm{X}}, \mathrm{NH}_{3}, \mathrm{PPM}$, and $\mathrm{SO}_{2}$ );

- The selected policy option, or level of ambition to improve air quality, in terms of emission reductions: in this paper we refer to four of the policies considered in the Air Quality Package Review (Amann et al., 2014), that will be expanded on at a later stage.

SHERPA is based on spatially dependent coefficients but it is not our place here to discuss the results on every grid cell. Instead, we restricted our uncertainty and sensitivity analysis to specific locations, namely four cities: Helsinki, Constanța, London, and Milan. These cities have been selected as they represent examples of different EU meteorological and emission inventory conditions. As stated, in this paper we refer to the SHERPA model linking emission reduction scenarios to average yearly/annual concentrations of PM2.5.

\subsection{Uncertainties of the SHERPA coefficients}

The implementation of SHERPA is based on data produced by the CHIMERE CTM model (Menut et al., 2013). In particular, the CTM has been used across the whole European territory with a spatial resolution of roughly $7 \times 7 \mathrm{~km}^{2}$. The anthropogenic emissions underlying the model simulations are based on the MACC-TNO emission inventory (Monitoring Atmospheric Composition and Climate data, produced by the TNO research institute) (Kuenen et al., 2014), with residential sector emissions modified to account for the enhanced wood consumption at extremely low temperatures (Terrenoire et al., 2015). The meteorological input data is based on IFS (Integrated Forecasting System from the European Centre for Medium-Range Weather Forecasts) for the year 2009. A set of CTM simulations in which emissions are reduced over the entire modelling domain are used to derive the $\alpha$ and $\omega$ coefficients. These are required in the simplified SHERPA equation, for each grid cell and precursor. An additional set of simulations, with reductions over specific areas, provide data for the method's validation. More details on the whole procedure can be found in Thunis et al. (2016), and Pisoni et al. (2017). The identification process previously described led to assign a normal distribution to each $\alpha$ and $\omega$ coefficients. The characteristics of the normal distributions are reported in Table 1 for the cities studied.

\subsection{Uncertainties of the emissions and policies}

The emissions of $\mathrm{NO}_{\mathrm{X}}, \mathrm{NH}_{3}, \mathrm{PPM}$, and $\mathrm{SO}_{2}$, expressed in Ktons/year are assumed to be uniformly distributed around their respective nominal value, as no better assumptions can be derived on the shape of these Probability Distribution Functions. While nominal values come from the considered SHERPA emission inventory, uncertainties (range of variability) have been derived from scientific literature (Nielsen et al., 2014; Kuenen et al., 2014), and considered the same for all city cases. For example: $\mathrm{NO}_{\mathrm{X}}$ varies of an amount of $\pm 30 \%$ around its nominal value as indicated in Table 2 .

Finally, four policy options have been selected for this study, to

Table 2

Precursors uniform distribution range.

\begin{tabular}{ll}
\hline Precursors & Range \\
\hline $\mathrm{NO}_{\mathbf{X}}$ & {$[-0.3,+0.3]$} \\
$\mathrm{NH}_{3}$ & {$[-0.5,+0.5]$} \\
$\mathrm{PPM}$ & {$[-0.5,+0.5]$} \\
$\mathrm{SO}_{2}$ & {$[-0.1,+0.1]$} \\
\hline
\end{tabular}


represent different levels of ambition in trying to improve air quality between the CLE (Current Legislation) and the MFR (Maximum Feasible Reductions ${ }^{3}$ ). In particular, the four policies considered in this study represent air quality improvement at 25\%, 50\%, 75\% (between CLE and MFR), and at $100 \%$ (that is to say, MFR). ${ }^{4}$ It is important to note that here we use an "EU wide" definition of CLE and MFR, so that the policy adhered to in this paper is the same for all the cities considered.

\section{Results and discussion}

\subsection{Uncertainty analysis results}

The uncertainty analysis is performed to evaluate the range of variability of the output (reduction of PM2.5 concentrations), due to the model input uncertainties. This requires propagation of the input uncertainties into the output of interest (i.e. yearly average concentrations of PM2.5). This propagation is performed using Monte Carlo simulations of SHERPA. For this purpose, a Monte Carlo sample of the input variables has been generated. This sample is a " $2 \mathrm{~N} \mathrm{x} \mathrm{d"} \mathrm{matrix}$ $(\mathrm{N}=1024, \mathrm{~d}=13$ ) each column containing the values of each input sampling from their probability density function (see Section 3). Each row of the matrix represents a set of model input values that is used to run SHERPA. Running SHERPA for each row of input values provide the vector of model responses with the size of $2 \mathrm{~N}=2048$. Note that, in reference to Eqs. (8) and (9), the sample is composed of the two input samples A and $\mathbf{B}$ each of size N. We grouped the uncertainty analysis on the basis of the four different policies selected, that is to say considering air quality improvement at $25 \%, 50 \%, 75 \%$, and $100 \%$ (as said, between Current LEgislation and Maximum Feasible Reductions).

Regarding the four cities of Helsinki, Constanța, London, and Milan (Fig. 2), we report, for each policy, the density distributions of the percentage change of concentration (with respect to the base case) as resulting from the input uncertainties. They were obtained, from the Monte Carlo simulations of SHERPA, by using the kernel density estimation algorithm of Botev et al. (2010). In particular, the x-axis represents the percentage change in concentrations of PM2.5 yearly average, and the y-axis represents the probability density value.

In general, we note that similar results are observed for the investigated cities. In particular, the more ambitious the policy applied, the more the estimated probability density function is shifted towards higher pollutant concentration reduction (Fig. 2).

This shift is accompanied with an increase of the predicted uncertainty ranges which means that the more ambitious is the chosen policy, the less certain is the predicted PM2.5 yearly average. Notably, the distributions spread over a larger range (i.e. $[15,80] \%$ ) for the city of Milan. This indicates that Milan represents the highest potential of pollutant concentration reduction. This makes sense as the area of Milan is known to be more polluted than the other three cities mentioned.

In the case of Milan (Fig. 2d), we can infer that by applying policy $25 \%$, according to SHERPA, one would expect an abatement of between $[15,50] \%$ while by applying a more ambitious policy, say the $50 \%$ policy, the predicted reduction lies within $[18,60] \%$. Given that the

\footnotetext{
${ }^{3}$ The 'Maximum feasible reduction' (MFR) scenario simulates how much of the emissions of the various substances could be further reduced beyond the current legislation, through the application of all available technical measures, without changes in the energy structures and without behavioral changes of consumers. The MFR considered is defined as in Amann et al. (2013).

${ }^{4}$ The percentage reductions considered in this paper have been chosen to analyze the full span of possible variability of the model, so to test thoroughly its behavior. Also, note that $100 \%$ of MFR does not mean a reduction of $100 \%$ of a pollutant, but of the total amount of emissions that can be reduced if all available technologies would be chosen (100\% MFR i.e. means 59\% emission reductions for PPM, 34\% reductions for ammonia, etc.). Finally, "MFR extreme cases" ( $75 \%$ or $100 \%$ reductions) allow also to evaluate how the model would behave in case of "energy structure" changes, that are not included in the MFR and that could potentially strongly reduce emissions.
}

application of these two different policies, in an uncertain context, provides ranges that largely overlap, it may be questioned whether it is worthy applying a $50 \%$ policy (more ambitious and so much more expensive) instead of $25 \%$ policy. This overlapping of the estimated PDFs is concurrent with their flattening (and enlargement) which means that the predictions prove to be more uncertain with ambitious policies.

In the context of policy decision-making, we are facing this challenge in an uncertain framework. Because of the uncertainties attached to the model, there is no evidence that by choosing one policy over another that any significant improvement will be achieved when compared with the choice of a less ambitious policy. This is particularly relevant when the former is more expensive to apply than the latter. We can confidently say that, under the present circumstances, decision making suffers from a lack of robustness. Such a lack of robustness would not occur if we had obtained non-overlapping and narrower predicted uncertainties. Therefore, it is crucial to identify the uncertain inputs which are responsible for the predicted uncertainties. This would lead the way to where to place future efforts in order to reduce uncertainties in SHERPA's predictions and eventually be able to make a reliable choice from the different policy options.

The uncertainty analysis is not able to apportion the total output variability to the different variable input factors (input and model coefficient). To do so, it is necessary to perform sensitivity analysis, which quantifies output uncertainty caused by the different model inputs, and identifies the main sources of uncertainty linked to higher sensitivity indices.

\subsection{Sensitivity indices}

An initial sensitivity analysis is made considering all together the 13 uncertain variables, namely the four $\alpha$ coefficients, the four $\omega$ coefficients, the four emissions $\left(\mathrm{NO}_{\mathrm{X}}, \mathrm{NH}_{3}, \mathrm{PPM}\right.$, and $\left.\mathrm{SO}_{2}\right)$, and the policy options. This was achieved with an extra cost of $13 \times 1024$ runs of the SHERPA model accordingly with the methods described at the end of Section 2.2 (see Eqs. (8) and (9)). For all inputs explored, results in terms of first-order indices and total order indices are very similar, proving that interactions among model inputs are virtually absent. As a result, we only report and discuss the total order indices $T_{i}$ (particularly relevant with regards to sensitivity analysis).

The total sensitivity indices estimates are reported in Fig. 3 for the first analysis (with uncertain policy options) and Fig. 4 (for a fixed policy choice). More in details, the different terms in the x-axis represent:

$$
\begin{aligned}
& \text { - } \omega_{-}\left\{\mathrm{NO}_{\mathrm{x}}, \mathrm{NH}_{3}, \mathrm{ppm}, \mathrm{SO}_{2}\right\} \text { and } \alpha_{-}\left\{\mathrm{NO}_{\mathrm{x}}, \mathrm{NH}_{3}, \mathrm{ppm}, \mathrm{SO}_{2}\right\} \text { : the model } \\
& \text { coefficient; } \\
& \text { - } \mathrm{NO}_{\mathrm{x}}, \mathrm{NH}_{3}, \mathrm{PPM} \text {, and } \mathrm{SO}_{2} \text { : the input of the model (emission); } \\
& \text { - 'Policy': the selected 'ambition' level for the analysis; }
\end{aligned}
$$

while in the y-axis the total sensitivity index values are indicated.

The results for all cities show the input "policy option" as a predominant contributor to the accuracy of the model results. This is definitely the case for the most important source of uncertainty in three cases (Helsinki, Constanța, and London), and the second most important input for Milan (about $44 \%$ of output uncertainty postulated by the policy option whereas the PPM has a total sensitivity index of 53\%). This means that the choice of policy heavily influences the predicted concentration reductions.

In this case, the first action is on the policy makers who should discuss upon what is the best policy to put in place. Later on, once the policy has been agreed upon, and the uncertainty reduced, the focus could be moved to the other inputs. However, the city of Milan is rather particular, because of the considerably high emissions of PPMs, even of more importance than the choice of policy. This fact suggests that it would be wise not to strictly embark in discussions about the optimal 
(a)

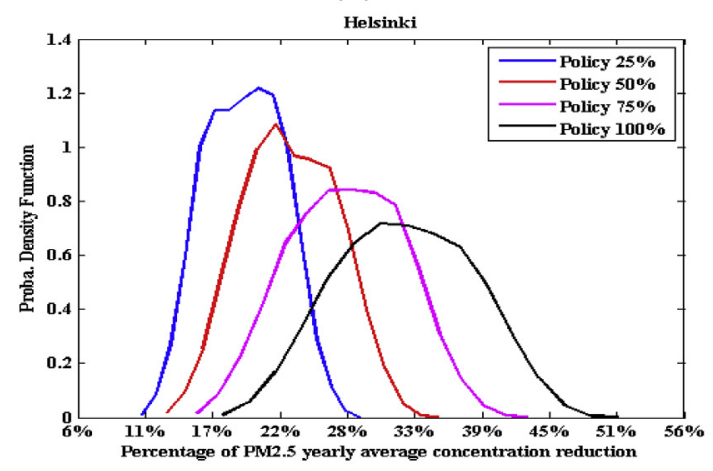

(c)

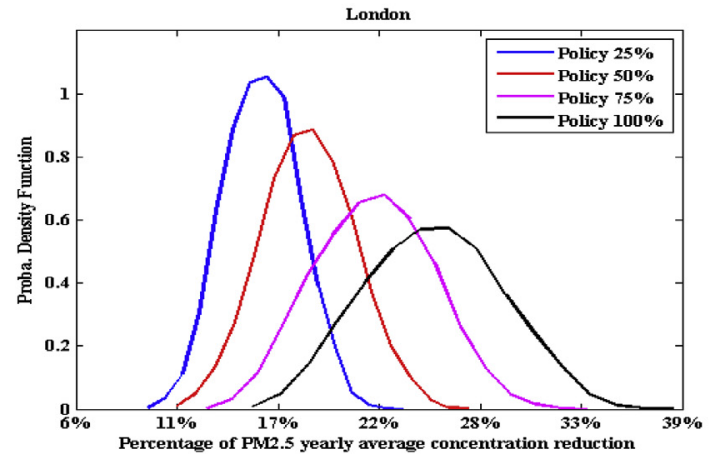

(b)

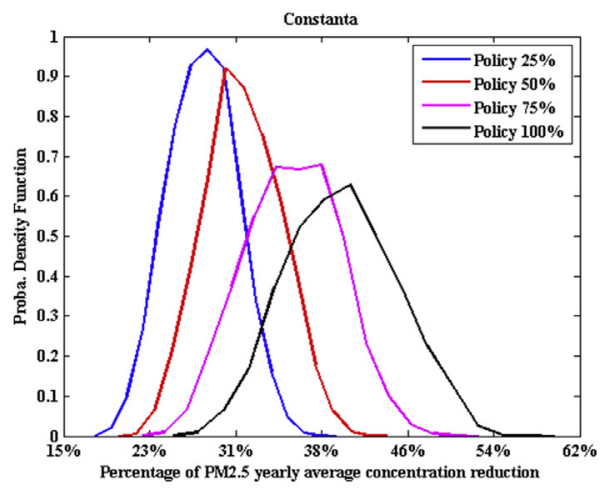

(d)

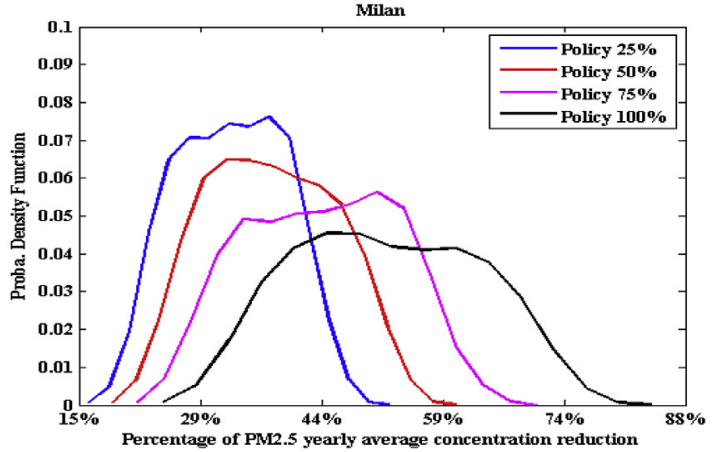

Fig. 2. Estimated probability density functions of predicted PM2.5 yearly average concentration reduction for different policies and different cities.

(a)

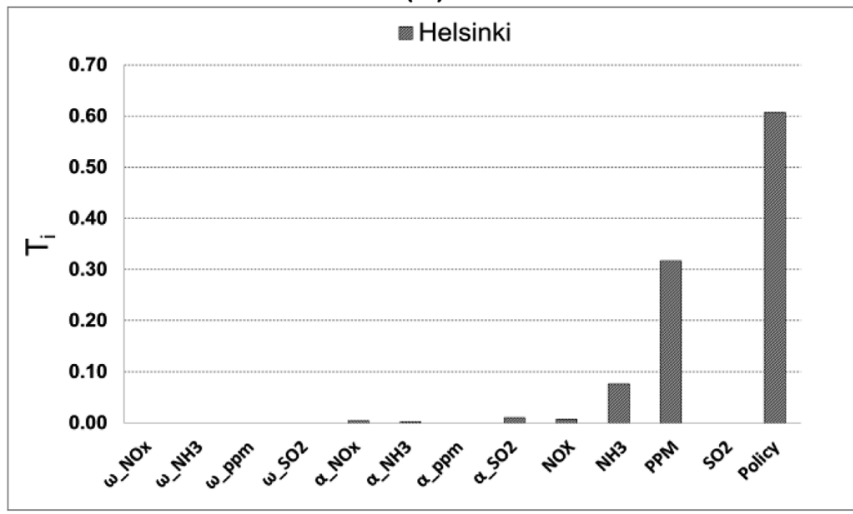

(c)

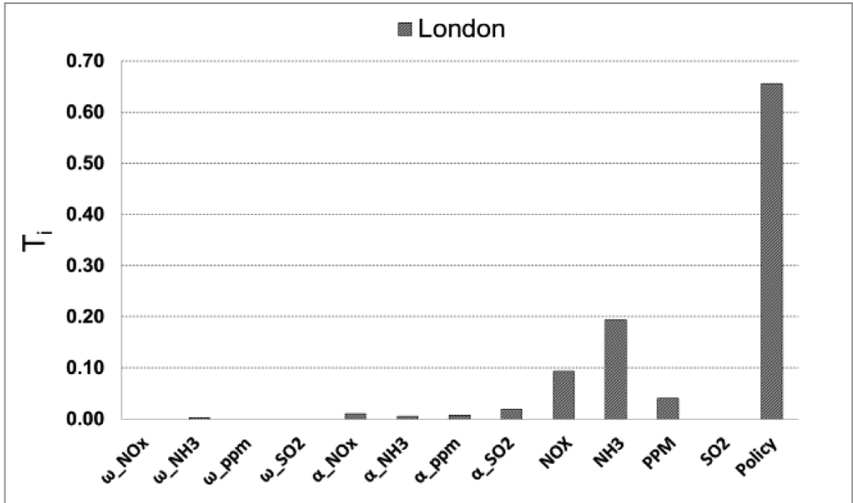

(b)

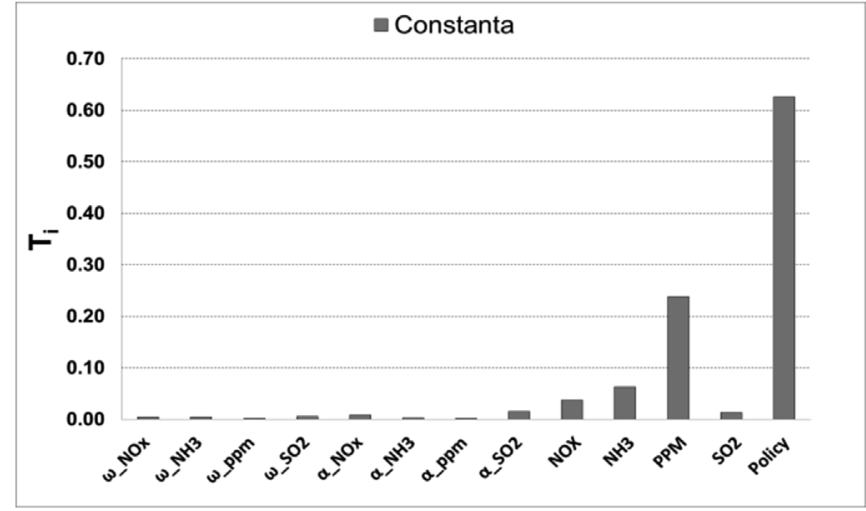

(d)

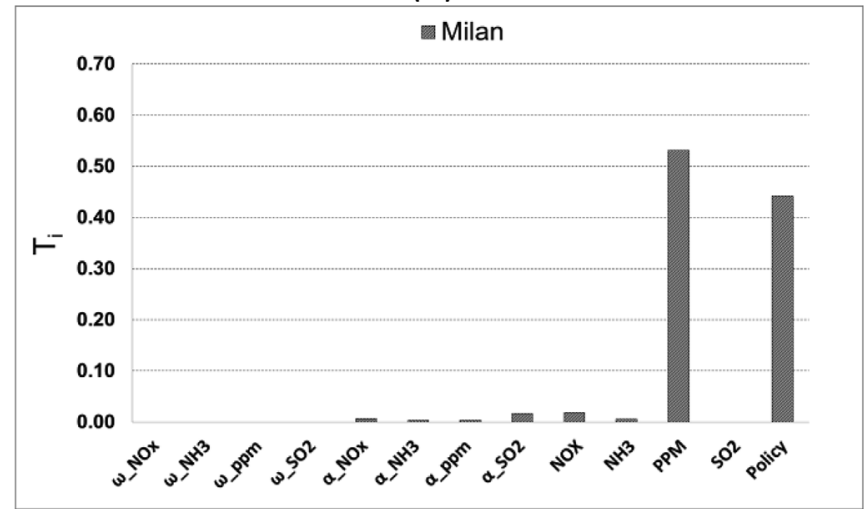

Fig. 3. Total sensitivity indices of model coefficients, emissions, and policy option. 
(a)

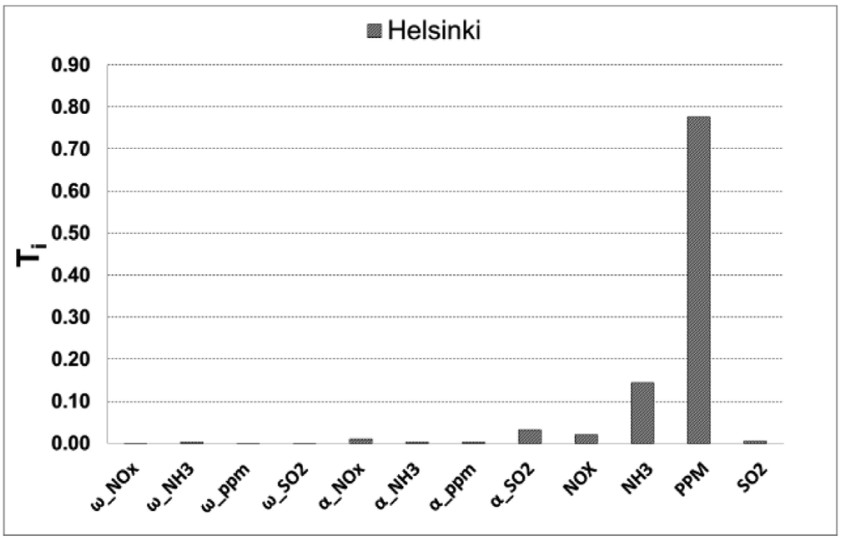

(c)

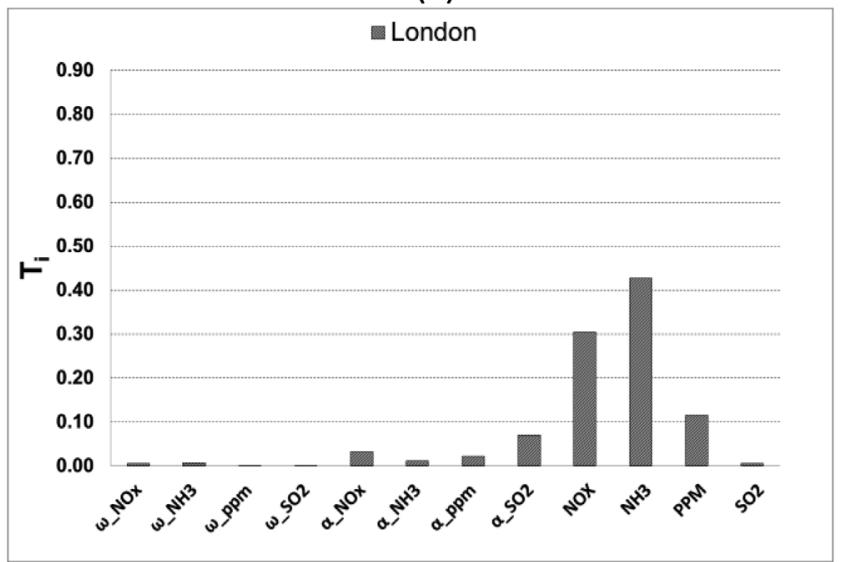

(b)

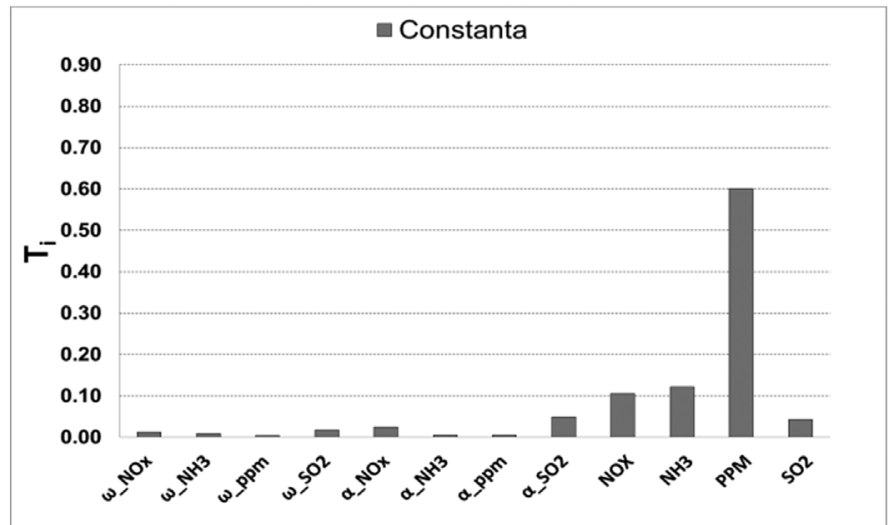

(d)

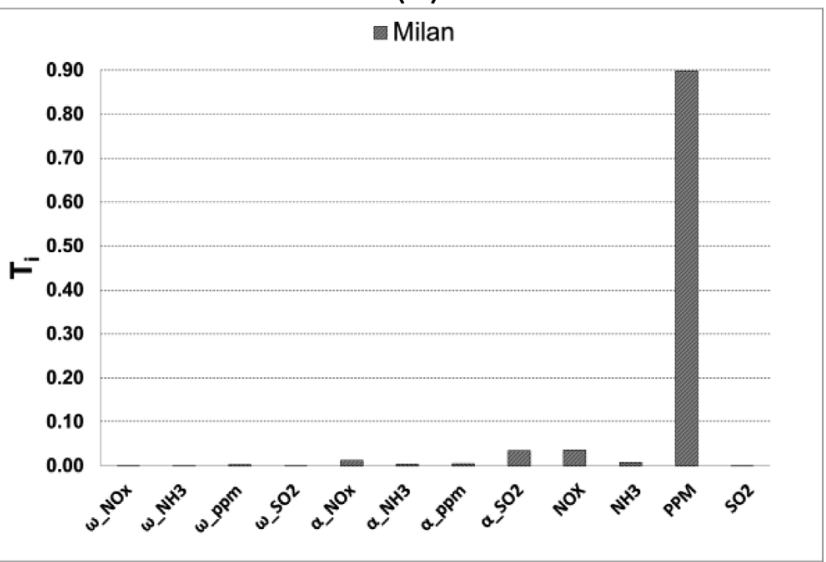

Fig. 4. Total sensitivity indices of model coefficients and emissions for policy $50 \%$.

policy to pursue, but, first (or at the same time/simultaneously), to try to improve the evidence based on the emissions of PPMs.

In all cases, the inputs related to $\alpha$ and $\omega$ have quite a negligible influence on concentration reductions. This means that the modeler and the analyst do not need to spend time and efforts in further reducing the uncertainty of such inputs, because the value of the concentration reductions will not show any noticeable improvement in terms of accuracy and the results of such efforts would not be useful for the policy.

In the second analysis, we assume that a given policy has been selected (say, the one at $50 \%$ air quality improvement). As a result, being fixed, this model input is no longer a source of uncertainty. As a second step, a new sensitivity analysis has been carried out in order to see which inputs, among the 12 remaining, are those which mostly contribute to the uncertainty in the concentration reduction. Note that this has been achieved without additional runs of the model. The results are reported in Fig. 4.

First we note that except for the city of London (Fig. 4c), the most significant input is by far the emission of PPMs. It explains around $80 \%$ of the total variance for the city Helsinki (Fig. 4a), 60\% for Constanța (Fig. 4b), and $90 \%$ of response variances for Milan (Fig. 4d). In the latter case, a more accurate estimation of the emission concentration of PPM is crucial. The second most significant uncertain input is the emission concentration of $\mathrm{NH}_{3}$ especially with relation to the cities of Helsinki and Constanța. In the case of Constanța, the uncertainty on the emission of $\mathrm{NO}_{\mathrm{X}}$ contributes equally with $\mathrm{NH}_{3}$ (about $10 \%$ ). The effects of the other inputs are negligible for these three cities. Note that when we consider $\mathrm{NH}_{3}$ (as also the other precursor emissions), we are not considering the emission of the city as such, but the emission of the whole domain 'weighted' as shown in Equation (1). So when referring to the uncertainty of one precursor, we are referring to the 'general' uncertainty of the emission inventory.

In the case of London (Fig. 4c), four sources of uncertainty are responsible for the response total variance, namely, $\mathrm{NH}_{3}, \mathrm{NO}_{\mathrm{X}}, \mathrm{PPM}$, and $\alpha_{-} \mathrm{SO}_{2}$ (by order of importance). We find that emission of $\mathrm{NO}_{\mathrm{X}}$ and emission of $\mathrm{NH}_{3}$ are the most important inputs (sensitivity indices are 0.30 and 0.43 respectively). This means that these two model inputs are, now, the main contributors to the model output uncertainty for the city of London. Therefore, if we want to improve the accuracy of concentration reductions, and also in this way, the evidence for policydecision making, we should concert our efforts towards reducing the uncertainty on the emissions of $\mathrm{NO}_{\mathrm{X}}$ and $\mathrm{NH}_{3}$. PPM and $\alpha_{-} \mathrm{SO}_{2}$ follow, and contribute as a whole to explain approximately $18 \%$ of model output uncertainty.

Apart for $\alpha_{-} \mathrm{SO}_{2}$, the model coefficients ( $\alpha$ and $\omega$ ) and emission $\mathrm{SO}_{2}$ are less influential contributors, even if, in general, the $\alpha$ coefficients appear as being more significant than the $\omega$ ones. Thus, actions related to these model inputs would not contribute significantly to concentration reductions. However, the relative importance of $\alpha_{-} \mathrm{SO}_{2}$ (in the case, not given here, where only $\alpha$ and $\omega$ uncertainty are considered) also indicates that the modeler should pay attention to the coefficient $\alpha_{-} \mathrm{SO}_{2}$ linking emissions of $\mathrm{SO}_{2}$ (usually point sources) to concentrations. The $\alpha_{-} \mathrm{SO}_{2}$ coefficient is currently estimated but without specifying the height in which emissions of $\mathrm{SO}_{2}$ occur. Indeed, it is the authors' belief that the model could be improved by taking the vertical level (height) of $\mathrm{SO}_{2}$ emission into account. This is not the case in the current version of SHERPA. 


\section{Conclusions}

In this paper, we address the application of uncertainty and sensitivity analysis techniques on a simplified air quality model. As, in Europe, we are moving to a situation in which exceedances of air quality legislation thresholds are mainly measured in specific regions or cities, the focus of the paper has been on performing uncertainty and sensitivity analysis on a modelling tool specifically designed for these geographical scales. The considered modelling tool, SHERPA, has been developed to support regional/local decision makers to design plans for better air quality. In particular, SHERPA is used in this paper to predict air quality improvement linked to emission reduction scenarios. The SHERPA model output (in terms of yearly concentrations of PM2.5) has been evaluated through uncertainty analysis (to quantify variability in the model output) and sensitivity analysis (to identify the most influential inputs) with respect to four EU cities (Helsinki, Constanța, London, and Milan) representative of different EU meteorological and emission inventory conditions.

The UA-SA was performed taking into consideration uncertainties in the SHERPA model coefficients, in the emissions of precursors of PM2.5 concentrations, and considering four alternative policy options. Through the UA, it is found that the model response uncertainty in terms of PM2.5 concentration abatement is substantially high for the cities in question. In this framework, sensitivity analysis can help to identify the uncertain inputs responsible for high uncertainty in the prediction for PM2.5 concentration abatement. Such information is crucial for future research purposes.

The SA shows that the most influential inputs are by far the policy selection (in this case, the level of ambition to be considered in the design of the air quality plan) and the emissions, in particular of PPM, $\mathrm{NO}_{\mathrm{X}}$, and $\mathrm{NH}_{3}$. The model coefficients ( $\alpha$ and $\omega$ ) are the least influential inputs, even if the $\alpha$ coefficients are more significant than the $\omega$ ones. In particular, policy option is a key aspect. In fact, in 3 out of 4 cases (Helsinki, Constanța, and London) the choice of policy has the highest sensitivity index, showing that the impact of this variable is more influential than all other model inputs. As a result this means that, in using the model, the first action should be for the policy makers, who should discuss upon what is the best policy to put in place. Later on, once the policy has been agreed, discussion could move on as to how to reduce uncertainty of the input and model coefficients, while considering the most relevant ones (identified by significant sensitivity indices). In Milan, contrary to the other cities, the uncertainty in the emissions of PPMs is the main contributor to the accuracy of the model results. For this city, the suggestion is to spend resources in order to obtain better knowledge of PPMs emissions, before (or at the same time) moving in the policy arena context. All other sources of uncertainty are quite irrelevant with regards to Milan.

\section{Acknowledgments}

The Authors would like to acknowledge Laurence Rouil, Augustin Colette and Bertrand Bessagnet (INERIS) for performing the CHIMERE simulations used in this paper to train and validate the Source Receptor Relationships, and Alain Clappier (University of Strasbourg) that contributed to the SHERPA model design and implementation.

\section{References}

Albrecht, D., Mara, T.A., Pisoni, E., Rosati, R., Tarantola, S., 2018. Sensitivity analysis of the SHERPA air quality model. JRC Technical Report (in preparation).

Amann, M., Bertok, I., Borken-Kleefeld, J., Cofala, J., Heyes, C., Höglund-Isaksson, L. Klimont, Z., Nguyen, B., Posch, M., Rafaj, P., Sandler, R., Schöpp, W., Wagner, F., Winiwarter, W., 2011. Cost-effective control of air quality and greenhouse gases in Europe: modeling and policy applications. Environ. Model. Software 26 (12), 1489-1501.

Amann, et al., 2013. Policy Scenarios for the Revision of the Thematic Strategy on Air Pollution. TSAP Report \#10. IIAS Tecchnical Report.

Amann, M., Borken-Kleefeld, J., Cofala, J., Hettelingh, J.-P., Heyes, C., Höglund-Isaksson,
L., Holland, M., Kiesewetter, G., Klimont, Z., Rafaj, P., Posch, M., Sandler, R., Schöpp, W., Wagner, F., Winiwarter, W., 2014. The Final Policy Scenarios of the EU Clean Air Policy Package. TSAP Report \#11, IIASA Technical Report.

Bessagnet, B., Pirovano, G., Mircea, M., Aulinger, A., Calori, G., Ciarelli, G., Manders, A., Stern, R., Tsyro, S., Cuvelier, C., Thunis, P., García Vivanco, M., Pay, M.-T., Colette, A., Couvidat, F., Meleux, F., Rouill, L., Ung, A., Aksoyoglu, S., Baldasano, J.M., Bieser, J., Briganti, G., Cappelletti, A., D'Isidoro, M., Finardi, S., Fagerli, H., Kranenburg, R., Silibello, C., Carnevale, C., Dupont, J.-C., Gonzalez, L., Menut, L., Prévôt, A.S.H., Roberts, P., White, L., 2016. Presentation of the EURODELTA III intercomparison exercise - evaluation of the chemistry transport models' performance on criteria pollutants and joint analysis with meteorology. Atmos. Chem. Phys. 16, $12667-12701$.

Blatman, G., Sudret, B., 2010. Efficient computation of global sensitivity indices using sparse polynomial chaos expansions. Reliab. Eng. Syst. Saf. 95, 1216-1229.

Borgonovo, E., 2007. A new uncertainty importance measure. Reliab. Eng. Syst. Saf. 92 (6), 771-784.

Botev, Z.I., Grotowski, J.F., Kroese, D.P., 2010. Kernel density estimation via diffusion. Ann. Stat. 38 (5), 2916-2957.

Buzzard, G.T., Xiu, D., 2011. Variance-based global sensitivity analysis via sparse-grid interpolation and curbature. Commun. Comput. Phys. 9, 542-567.

Campolongo, F., Saltelli, A., 1997. Sensitivity analysis of an environmental model: an application of different analysis methods. Reliab. Eng. Syst. Saf. 57 (1), 49-69.

Carnevale, C., Finzi, G., Pisoni, E., Volta, M., Guariso, G., Gianfreda, R., Maffeis, G., Thunis, P., White, L., Triacchini, G., 2012. An integrated assessment tool to define effective air quality policies at regional scale. Environ. Model. Software 38, 306-315.

Carnevale, C., Finzi, G., Pederzoli, A., Turrini, E., Volta, M., Guariso, G., Gianfreda, R., Maffeis, G., Pisoni, E., Thunis, P., Markl-Hummel, L., Blond, N., Clappier, A., Dujardin, V., Weber, C., Perron, G., 2014. Exploring trade-offs between air pollutants through an integrated assessment model. Sci. Total Environ. 481, 7-16.

Clappier, A., Pisoni, E., Thunis, P., 2015. A new approach to design source-receptor relationships for air quality modelling. Environ. Model. Software 74, 66-74.

Cuvelier, C., Thunis, P., Vautard, R., Amann, M., Bessagnet, B., Bedogni, M., Berkowicz, R., Brandt, J., Brocheton, F., Builtjes, P., Carnavale, C., Coppalle, A., Denby, B., Douros, J., Graf, A., Hellmuth, O., Hodzic, A., Honoré, C., Jonson, J., Kerschbaumer, A., de Leeuw, F., Minguzzi, E., Moussiopoulos, N., Pertot, C., Peuch, V.H., Pirovano, G., Rouil, L., Sauter, F., Schaap, M., Stern, R., Tarrason, L., Vignati, E., Volta, M., White, L., Wind, P., Zuber, A., 2007. CityDelta: a model intercomparison study to explore the impact of emission reductions in European cities in 2010. Atmos. Environ. 41, (1), 189-207.

D'Elia, I., Bencardino, M., Ciancarella, L., Contaldi, M., Vialetto, G., 2009. Technical and Non-Technical Measures for air pollution emission reduction: the integrated assessment of the regional Air Quality Management Plans through the Italian national model. Atmos. Environ. 43 (39), 6182-6189.

Dunker, A.M., Yarwood, G., Ortmann, J.P., Wilson, G.M., 2002. The decoupled direct method for sensitivity analysis in a three-dimensional air quality model implementation, accuracy, and efficiency. Environ. Sci. Technol. 36, 2965-2976.

EEA, European Environmental Agency, 2017. Air Quality in Europe - 2017 Report.

Homma, T., Saltelli, A., 1996. Importance measures in global sensitivity analysis of nonlinear models. Reliab. Eng. Syst. Saf. 52 (1), 1-17.

Kelly, J.T., Baker, K.R., Napelenok, S.L., Roselle, S.J., 2015. Examining single-source secondary impacts estimated from brute-force, decoupled direct method, and advanced plume treatment approaches. Atmos. Environ. 111, 10-19.

Kiesewetter, G., Schoepp, W., Heyes, C., Amann, M., 2015. Modelling PM 2.5 impact indicators in Europe: health effects and legal compliance. Environ. Model. Software 74, 201-211.

Kucherenko, S., Tarantola, S., Annoni, P., 2012. Estimation of global sensitivity indices for models with dependent variables. Comput. Phys. Commun. 183 (4), 937-946.

Kuenen, J.J.P., Visschedijk, A.J.H., Jozwicka, M., Denier Van Der Gon, H.A.C., 2014. TNO-MACC_II emission inventory; a multi-year (2003-2009) consistent high-resolution European emission inventory for air quality modelling. Atmos. Chem. Phys. 14 (20), 10963-10976.

Liu, Q., Homma, T., 2009. A new computational method of a moment-independent uncertainty importance measure. Reliab. Eng. Syst. Saf. 94 (7), 1205-1211.

Mailler, S., Menut, L., Khvorostyanov, D., Valari, M., Couvidat, F., Siour, G., Turquety, S., Briant, R., Tuccella, P., Bessagnet, B., Colette, A., Létinois, L., Meleux, F., 2017. CHIMERE-2017: from urban to hemispheric chemistry-transport modeling. Geosci. Model Dev. Discuss. (GMDD) 10, 2397-2423.

Mara, T.A., Tarantola, S., 2012. Variance-based sensitivity indices for models with dependent inputs. Reliab. Eng. Syst. Saf. 107, 115-121.

Menut, L., Bessagnet, B., Khvorostyanov, D., Beekmann, M., Blond, N., Colette, A., Coll, I., Curci, G., Foret, G., Hodzic, A., Mailler, S., Meleux, F., Monge, J.-L., Pison, I., Siour, G., Turquety, S., Valari, M., Vautard, R., Vivanco, M.G., 2013. CHIMERE 2013: a model for regional atmospheric composition modelling. Geosci. Model Dev. (GMD) 6, 981-1028 doi:10.5194.

Nielsen, O.K., Winther, M., Mikkelsen, M.H., Hoffmann, L., Nielsen, M., Gyldenkærne, S., Fauser, P., Plejdrup, M.S., Albrektsen, R., Hjelgaard, K., Bruun, H.G., 2014. Annual Danish Informative Inventory Report to UNECE. Emission Inventories from the Base Year of the Protocols to Year 2012. Aarhus University, pp. 759. Scientific Report from DCE - Danish Centre for Environment and Energy No. 94. http://dce2.au.dk/pub/ SR94.pdf (Last Access June 2017).

Pernigotti, D., Thunis, P., Cuvelier, C., Georgieva, E., Gsella, A., De Meij, A., Pirovano, G., Balzarini, A., Riva, G.M., Carnevale, C., Pisoni, E., Volta, M., Bessagnet, B., Kerschbaumer, A., Viaene, P., De Ridder, K., Nyiri, A., Wind, P., 2013. POMI: a model inter-comparison exercise over the Po Valley. Air Qual. Atmos. \& Health 6 (4), 701-715.

Pisoni, E., Carnevale, C., Volta, M., 2010. Sensitivity to spatial resolution of modeling 
systems designing air quality control policies. Environ. Model. Software 25 (1), $66-73$.

Pisoni, E., Clappier, A., Degraeuwe, B., Thunis, P., 2017. Adding spatial flexibility to source-receptor relationships for air quality modeling. Environ. Model. Software 90, 68-77.

Plischke, E., Borgonovo, E., Smith, C.L., 2013. Global sensitivity measures from given data. Eur. J. Oper. Res. 226 (3), 536-550.

Saltelli, A., 2002. Making best use of model evaluations to compute sensitivity indices. Comput. Phys. Commun. 145, 280-297.

Saltelli, A., Annoni, P., 2010. How to avoid a perfunctory sensitivity analysis. Environ. Model. Software 25 (12), 1508-1517.

Saltelli, A., Tarantola, S., Chan, K.P.S., 1999. A quantitative model-independent method for global sensitivity analysis of model output. Technometrics 41, 39-56.

Saltelli, A., Ratto, M., Andres, T., Campolongo, F., Cariboni, J., Gatelli, D., Saisana, M., Tarantola, S., 2008. Global Sensitivity Analysis: the Primer. John Wiley \& Sons.

Saltelli, A., Annoni, P., Azzini, I., Campolongo, F., Ratto, M., Tarantola, S., 2010. Variance based sensitivity analysis of model output. Design and estimator for the total sensitivity index. Comput. Phys. Commun. 181 (2), 259-270.

Sandu, A., Daescu, D.N., Carmichael, G.R., 2003. Direct and adjoint sensitivity analysis of chemical kinetic systems with KPP: Part I-theory and software tools. Atmos. Environ. 37, 5083-5096.

Schaap, M., Cuvelier, C., Hendriks, C., Bessagnet, B., Baldasano, J.M., Colette, A., Thunis, P., Karam, D., Fagerli, H., Graff, A., Kranenburg, R., Nyiri, A., Pay, M.T., Rouïl, L.,
Schulz, M., Simpson, D., Stern, R., Terrenoire, E., Wind, P., 2015. Performance of European chemistry transport models as function of horizontal resolution. Atmos. Environ. 112, 90-105.

Shao, Q., Younes, A., Fahs, A., Mara, T.A., 2017. Bayesian sparse polynomial chaos expansion for global sensitivity analysis. Comput. Meth. Appl. Mech. Eng. 318, 474-496.

Sobol, I.M., 1993. Sensitivity estimates for nonlinear mathematical models. Math. Model. Comput. Exp. 1 (4), 407-414.

Sobol, I.M., 2001. Global sensitivity indices for nonlinear mathematical models and their Monte Carlo estimates. Math. Comput. Simulat. 55 (1), 271-280.

Tarantola, S., Giglioli, N., Jesinghaus, J., Saltelli, A., 2002. Can global sensitivity analysis steer the implementation of models for environmental assessments and decisionmaking? Stoch. Environ. Res. Risk Assess. 16 (1), 63-76.

Terrenoire, E., Bassagnet, B., Rouill, L., Tognet, F., Pirovano, G., Létinois, L., Beauchamp, M., Colette, A., Thunis, P., Amann, M., Menut, L., 2015. High-resolution air quality simulation over Europe with the chemistry transport model CHIMERE. Geosci. Model Dev. (GMD) 8 (1), 21-42.

Thunis, P., Pisoni, E., Degraeuwe, B., Kranenburg, R., Schaap, M., Clappier, A., 2015. Dynamic evaluation of air quality models over European regions. Atmos. Environ. 111, 185-194.

Thunis, P., Degraeuwe, B., Pisoni, E., Ferrari, F., Clappier, A., 2016. On the design and assessment of regional air quality plans: the SHERPA approach. J. Environ. Manag. 183, 952-958. 